\title{
Etiology of Diarrhoea with Reference to Multiple Drug Resistant Enteric Bacterial Pathogens
}

\author{
Sirjana Devi Shrestha ${ }^{1-2}$, Sarala Malla ${ }^{2}$ and Shital Raj Basnyat ${ }^{1}$ \\ ${ }^{l}$ Central Department of Microbiology, Tribhuwan University, \\ Kirtipur, Kathmandu, Nepal \\ ${ }^{2}$ National Public Health Laboratory, Kathmandu, Nepal
}

\begin{abstract}
A total of 340 stool samples were processed and studied from both sexes including all ages of patients. Association of enteropathogens between male and female was not statistically significant. Incidence of diarrhoea (28.23\%) as well as prevalence of enteropathogens $(34.31 \%)$ was found highest in the age group (20-30) years. The highest prevalence of enteropathogens (44.87\%) was found in August. Of the total isolated enteropathogens, Vibrio cholerae $\mathrm{O} 1$ was observed in $51.96 \%$ followed by Shigella $(18.6 \%)$ and Salmonella $(8.82 \%)$ and parasites were also detected from $20.58 \%$ samples. All isolated V. cholerae O1 were El Tor, Inaba. Among Shigella, majority of isolates were S. flexneri. Among Salmonella, S. typhi, S. typhimurium and Salmonella spp. (polyvalent A-S positive) were identified. Entamoeba histolytica, Girdia lamblia, Ascaris lumbricoides and Trichuris trichiura were isolated among parasites. All isolated $(100 \%) V$. cholerae O1 were resistant to nalidixic acid and cotrimoxazole, whereas $68 \% ; 63 \% ; 53 \% ; 37 \%$ and $11 \%$ Shigella were resistant to nalidixic acid, ampicillin, cotrimoxazol, mecillinam and ciprofloxacin respectively. Similarly, $55.5 \% ; 44.4 \%$ and $11.1 \%$ Salmonella were resistant to nalidixic acid; ampicillin and cotrimoxazole respectively. All $V$. cholerae strains, 10 strains of Shigella and 2 strains of Salmonella were found multi drug resistant (MDR). The clinical history of the positive cases revealed that abdominal pain, fever, vomiting, dehydration and nausea were the symptoms of enteric infection.
\end{abstract}

Key words: antibiotics, isolates, enteropathogen, Inaba

\section{Introduction}

Severe occurrence of acute diarrhoea constitutes one of the commonest challenges faced by the medical personnel and emergency treatment with special concern in the developing world and mild nuisance in the developed world. Diarrhoeal episodes occur throughout the year with epidemics in rainy season (Shrestha 1994).

Diarrhoea is a clinical syndrome of diverse aetiology, in which the sufferer has frequent and watery bowel movements. This condition can be a symptom of injury, disease or foodborne illness and is usually accompanied by abdominal pain and often nausea and vomiting. Diarrhoea removes essential body fluids, salts and vital nutrients, which are necessary for life producing dehydration and malnutrition. If more than $10 \%$ of the body's fluid is lost death occurs. The most common enteropathogens found in all countries are rotavirus, enterotoxigenic Escherichia coli, Vibrio species, Salmonella species, Shigella species and Campylobacter (Sunoto 1982). Similarly, adenovirus; parasites like Cryptosporidium, Entamoeba and Giardia have also been shown to play a role in the epidemiology of diarrhoea especially in certain areas of the globe. Intestinal worms infect about $10 \%$ of the population of the developing world (WHO 2004).

Low socioeconomic status, illiteracy, poor standard of environmental hygiene, inaccessibility of safe drinking water, incorrect feeding practice, inadequate sanitation, infections and immunodeficiency are the predisposing factors for diarrhoea. According to WHO, around 4 billion cases of diarrhoea are recorded each year, leading to 2.2 million deaths, mostly among children under the age of five (15\% of all child deaths). The main cause of death from diarrhoea is dehydration, dysentery and malnutrition and other infections (WHO 1992).

In Nepal diarrhoeal disease is considered as one of the commonest health problems and cause of morbidity and mortality. Outbreaks of diarrhoeal diseases are mostly reported from April to September each year. In Nepal, owing to the low socioeconomic status and poor hygienic condition of the people, intestinal parasitic and bacterial infections constitute an important cause of morbidity and mortality 
(Sherchand et al. 1996). The case fatality rate in Nepal from diarrhoea is highest $(2 \%)$ in the age group of 1-4 and is lowest (1\%) among the infants (Bista et al. 1993). Improvement and control of environmental and behavioral facts may drastically reduce prevalence and impact of diseases.

Antibiotic therapy is not required in most acute diarrhoeal disorders (Wolf \& Giannella 1993). In these years many bacterial strains are found to be resistant to antibiotics which reflect the widespread misuse of antidiarrhoeal drugs. Each time the antibiotics are administered, the normal flora is also exposed. Sometimes they may also exhibit resistance to several antibiotics. In developing country like Nepal, the problem of misuse of drugs is very common either in the form of incomplete dosages, unnecessary usage or use of antibiotics as growth promoters in veterinary medicine.

\section{Materials and Methods}

All the laboratory works were conducted at the bacteriology section of National Public Health Laboratory, Teku from March to September 2005. During this period a total of 340 stool samples from diarrhoeal patients were collected and processed according to the standard laboratory methods.

Each patient suffering from diarrhoea was directly interviewed for his or her clinical history during sample collection. The stool sample requested for culture were collected in sterile clean container having tight fitting and leak proof lid. Fresh stool specimens were first examined macroscopically. Microscopic examination was conducted to know the presence of parasites. The stool samples from suspected cholera patients were subjected to hanging drop preparation. To detect the parasites, saline wet mount and iodine wet mount methods were performed.

\section{Isolation and identification of bacterial pathogens}

\section{Vibrio cholerae}

\section{Culture of specimens}

One milliliter of fresh stool sample was inoculated in $10 \mathrm{ml}$ alkaline peptone water (APW) for enrichment. Heavy inoculums of stool were inoculated on the plate of thiosulphate citrate bile salt sucrose (TCBS) medium.
After incubation for about 5 hours, a loopful sample from APW was also inoculated in second plate of TCBS and incubated aerobically at $37^{\circ} \mathrm{C}$ for 24 hours. After incubation, the culture plates were observed for growth of sucrose fermenting yellow, 2-3 $\mathrm{mm}$ in diameter, button shaped colonies. The isolated yellow colony was subcultured on NA plate and incubated for 24 hours at $37^{\circ} \mathrm{C}$. Further identification was done by NA plate culture.

Gram stain, different biochemical tests (oxidase test, catalase test, indole test, methyl red test, vogesproskaur test, citrate utilization test, oxidativefermentative test, sulphide, indole and motility test, triple sugar iron test, urea hydrolysis test, salt concentration tolerance test, string test); serotyping (by slide agglutination) and biotyping of Vibrio cholerae O1 (Haemagglutination of Chick RBC, haemolysis of sheep RBC, susceptibility to polymyxin B, VPReaction) were done for identification.

\section{For Salmonella and Shigella species Culture of specimens}

A large loopful of faeces was inoculated onto Salmonella-Shigella (SS) agar and MacConkey agar (MA) spreading an area one-fifth of the whole plate and several loopfuls into Selenite F (SF) broth. The loop was flamed, cooled and spreaded the primary inoculum on the remainder of the plate by semi-quantitative method. The plates and enrichment broth were incubated for 24 hours at $37^{\circ} \mathrm{C}$ temperature.

A loopful of suspension from SF broth was subcultured onto SS agar and incubated for 24 hours at $37^{\circ} \mathrm{C}$. Each pale non lactose fermenting isolated colony on primary SS agar and MA as well as sub-cultured SS agar plate was observed for regal characteristics and subcultured on NA. The NA plate was incubated at $37^{\circ} \mathrm{C}$ for 24 hours. From the NA plate further Gram staining, biochemical tests and serological tests were performed for the identification.

Gram stain; different biochemical tests (Oxidase test, Catalase test, Indole test, Methyl Red test, VogesProskaur test, Citrate Utilization test, OxidativeFermentative test, Sulphide, Indole and Motility test, Triple Sugar Iron test, Urea Hydrolysis test); serotyping ( by slide agglutination) were done for identification. 


\section{Antimicrobial susceptibility test}

Isolated colony of organisms was transferred to a test tube containing 4-5 ml nutrient broth and incubated at $37^{\circ} \mathrm{C}$ for 4 hours. After 4 hours, the tube was matched to the $0.5 \mathrm{McFarland}$ turbidity standard, it was then swabbed on Mueller-Hinton Agar plate with the help of sterile cotton swab and left for 15 minutes at room temperature for drying. With the help of flamed forceps, selected antibiotic discs were then placed on the inoculated plate, no closer than $15 \mathrm{~mm}$ from the edge of the plate and $25 \mathrm{~mm}$ apart from each other. Then the plate was allowed for 30 minutes at room temperature for diffusion and incubated at $37^{\circ} \mathrm{C}$ for 24 hours. After incubation, the zone of inhibition of test organism was measured and observed into sensitive, intermediate, and resistant categories by referring in an interpretative chart table supplied by the disc manufacturers.

\section{Results}

Out of 340 patients, 282 (82.94\%) were from Shukraraj Tropical Hospital and $58(17.05 \%)$ were from different localities of Kathmandu valley attending NPHL by requesting stool culture. Of the total patients, $58.52 \%$ were males and $41.47 \%$ were females. Ninty six patients belonged to the age group of 21-30 years.

Out of 102 enteropathogens detected, 60 (58.82\%) were from male patients and remaining $42(41.17 \%)$ were from female patients. There was no significant association of enteropathogens between male and female. During this study, patients of age group 21-30 years were frequently affected. The total enteropathogens observed in this group were $35(34.31 \%)$ and the age group 71-80 years had the least number of isolates, i.e., $2(1.96 \%)$.

Table 1. Distribution of enteropathogens during the study period

\begin{tabular}{lcc}
\hline Types of organisms & No. of organisms & Percentage \\
\hline Vibrio cholerae O1, EI Tor, Inaba & $\mathbf{5 3}$ & $\mathbf{5 1 . 9 6}$ \\
Shigella spp. & $\mathbf{1 9}$ & $\mathbf{1 8 . 6 2}$ \\
S. dysenteriae & $\mathbf{5}$ & $\mathbf{4 . 9}$ \\
S. flexneri & $\mathbf{1 0}$ & $\mathbf{9 . 8}$ \\
S. boydii & $\mathbf{3}$ & $\mathbf{2 . 9 4}$ \\
S. sonnei & $\mathbf{1}$ & $\mathbf{0 . 9 8}$ \\
Salmonella spp. & $\mathbf{9}$ & $\mathbf{8 . 8 2}$ \\
S. typhi & 2 & 1.96 \\
S. typhimurium & 2 & 1.96 \\
Salmonella spp. (Polyvalent A-S positive) & 5 & 4.9 \\
Parasites & $\mathbf{2 1}$ & $\mathbf{2 0 . 5 8}$ \\
Entamoeba histolytica & 12 & 11.76 \\
Giardia lamblia & 6 & 5.88 \\
Ascaris lumbricoides & 2 & 1.96 \\
Trichuris trichiura & 1 & 0.98 \\
Total & $\mathbf{1 0 2}$ & $\mathbf{1 0 0}$ \\
\hline
\end{tabular}

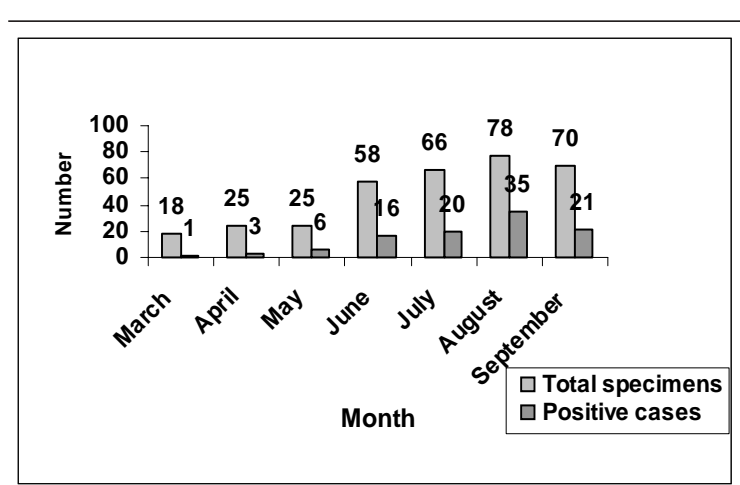

Fig. 1. Enteric pathogen positive cases during various months
The isolation of $V$. cholerae $\mathrm{O} 1$ was found to be in peak in August (45.28\%) and the incidence of Shigella was highest in July (31.57\%) and was seen in all 7 months of study period. The isolation rate of Salmonella was seen highest in June $(33.33 \%)$. Similarly, cases of parasites were highest (28.57\%) in August.

Clinical features induced by various enteric isolates indicated that abdominal pain was a common symptom among patients with enteropathogens. Fever was most frequently reported from the patients with salmonellosis $(66.6 \%)$ followed by shigellosis $(36.8 \%)$. Vomiting was reported from majority of the patients with $V$. cholerae 
O1 $(96.2 \%)$. Patients $(88.69 \%)$ with cholera had got the symptoms of dehydration, which is the prominent feature of this disease. and dehydrated cases were $44.44 \%$ and $36.84 \%$ among patients with Salmonella and Shigella infection respectively. Cholera patients
$(58.49 \%)$ and few patients with Shigella $(10.5 \%)$ and Salmonella (33.3\%) had got nausea also. Fever (9.52\%) and vomiting $(14.2 \%)$ were rarely reported from the patients during parasitic infection.

Table 2. Antibiotic susceptibility pattern of $V$. cholerae 01

\begin{tabular}{|c|c|c|c|c|c|c|c|c|c|c|c|c|c|c|}
\hline & \multicolumn{12}{|c|}{ Antibiotics used } & \multirow{2}{*}{\multicolumn{2}{|c|}{$\mathrm{T}$}} \\
\hline & \multicolumn{2}{|c|}{ Amp } & \multicolumn{2}{|c|}{$\mathrm{Cf}$} & \multicolumn{2}{|c|}{ NA } & \multicolumn{2}{|c|}{ Sxt } & \multicolumn{2}{|c|}{$\mathrm{E}$} & \multicolumn{2}{|c|}{$\mathrm{F}$} & & \\
\hline & $\mathrm{S}$ & $\mathrm{R}$ & $\mathrm{S}$ & $\mathrm{R}$ & $S$ & $\mathrm{R}$ & S & $\mathrm{R}$ & $S$ & $\mathrm{R}$ & S & $\mathrm{R}$ & $S$ & $\mathrm{R}$ \\
\hline Number & 53 & - & 53 & - & - & 53 & - & 53 & 53 & - & 8 & 45 & 53 & - \\
\hline Percentage & 100 & - & 100 & - & - & 100 & - & 100 & 100 & - & 15 & 84.9 & 100 & - \\
\hline
\end{tabular}

Table 3. Antibiotic susceptibility pattern of Shigella spp.

\begin{tabular}{|c|c|c|c|c|c|c|c|c|c|c|c|c|c|c|}
\hline & \multicolumn{12}{|c|}{ Antibiotics used } & \multirow{2}{*}{\multicolumn{2}{|c|}{ NA }} \\
\hline & & $\mathrm{mp}$ & & & $\mathrm{Cl}$ & & $\mathrm{C}$ & & $\mathrm{S}$ & & $\mathrm{M}$ & & & \\
\hline & $S$ & $\mathrm{R}$ & $\mathrm{S}$ & $\mathrm{R}$ & $S$ & $\mathrm{R}$ & $S$ & $\mathrm{R}$ & $\mathrm{S}$ & $\mathrm{R}$ & $S$ & $\mathrm{R}$ & $S$ & $\mathrm{R}$ \\
\hline Shigella No. & 7 & 12 & 19 & - & 19 & - & 17 & 2 & 6 & 11 & 12 & 7 & 6 & 13 \\
\hline spp. (19) (\%) & 36.8 & 63.2 & 100 & - & 100 & - & 89.5 & 10.5 & 35.3 & 64.7 & 63.2 & 36.8 & 31.6 & 68.4 \\
\hline
\end{tabular}

Table 4. Antimicrobial susceptibility pattern of Salmonella spp.

\begin{tabular}{|c|c|c|c|c|c|c|c|c|c|c|c|c|c|c|}
\hline \multirow[t]{3}{*}{$\begin{array}{c}\text { Types of } \\
\text { Salmonella }\end{array}$} & \multicolumn{8}{|c|}{ Antibiotics used } & & & & & & \\
\hline & \multicolumn{2}{|c|}{ Amp } & \multicolumn{2}{|c|}{$\mathrm{CRO}$} & \multicolumn{2}{|c|}{$\mathrm{C}$} & \multicolumn{2}{|l|}{$\mathrm{Cf}$} & \multicolumn{2}{|c|}{ Sxt } & \multicolumn{2}{|c|}{ NA } & \multicolumn{2}{|c|}{$\mathrm{T}$} \\
\hline & $\mathrm{S}$ & $\mathrm{R}$ & $\mathrm{S}$ & $\mathrm{R}$ & $\mathrm{S}$ & $\mathrm{R}$ & $\mathrm{S}$ & $\mathrm{R}$ & $\mathrm{S}$ & $\mathrm{R}$ & $\mathrm{S}$ & $\mathrm{R}$ & $\mathrm{S}$ & $\mathrm{R}$ \\
\hline S. typhi (2) & 2 & - & 2 & - & 2 & - & 2 & - & 2 & - & - & 2 & 2 & - \\
\hline S.typhimurium (2) & 1 & 1 & 2 & - & 2 & - & 2 & - & 2 & - & 1 & 1 & 2 & - \\
\hline $\begin{array}{l}\text { Salmonella spp. } \\
\text { (Polyvalent A-S positive) }\end{array}$ & 2 & 3 & 5 & - & 5 & - & 5 & - & 4 & 1 & 3 & 2 & 5 & - \\
\hline Total No. & 5 & 4 & 9 & - & 9 & - & 9 & - & 8 & 1 & 4 & 5 & 9 & - \\
\hline$\%$ & 55.5 & 44.4 & 100 & - & 100 & - & 100 & - & 88.8 & 11.1 & 44.4 & 55.5 & 100 & - \\
\hline
\end{tabular}

Table 5. Distribution of multiple drug resistant (MDR) enteric bacterial isolates

\begin{tabular}{|c|c|c|c|c|c|c|c|c|c|c|c|}
\hline \multirow{3}{*}{$\begin{array}{l}\text { Type and no. } \\
\text { of organisms }\end{array}$} & \multirow{3}{*}{$\begin{array}{l}\text { Sensitive to all } \\
\text { drugs tested }\end{array}$} & \multicolumn{8}{|c|}{ Drug resistance pattern } & \multirow{2}{*}{\multicolumn{2}{|c|}{$\begin{array}{l}\text { Total MDR } \\
\text { (i.e. resistant } \\
\text { to e" } 2 \text { drugs) } \\
\text { strains }\end{array}$}} \\
\hline & & \multicolumn{2}{|c|}{1 drug } & \multicolumn{2}{|c|}{2 drug } & \multicolumn{2}{|c|}{3 drug } & \multicolumn{2}{|c|}{$\begin{array}{l}\text { More than } \\
3 \text { drug }\end{array}$} & & \\
\hline & & No. & $\%$ & No. & $\%$ & No. & $\%$ & No. & $\%$ & No. & $\%$ \\
\hline V. cholerae (53) & - & - & - & 8 & $15.09 \%$ & 45 & $84.9 \%$ & - & - & 53 & 100 \\
\hline Shigella (19) & $4(21.05 \%)$ & 5 & 26.31 & 4 & $21.05 \%$ & 3 & $15.78 \%$ & 3 & $15.78 \%$ & 10 & 52.65 \\
\hline Salmonella (9) & $2(22.22 \%)$ & 5 & 545.55 & 1 & $11.11 \%$ & 1 & $11.11 \%$ & - & - & 2 & 22.22 \\
\hline Total (81) & $6(7.4 \%)$ & 10 & $12.34 \%$ & 13 & $16.04 \%$ & 49 & $60.49 \%$ & 3 & $3.7 \%$ & 65 & $80.24 \%$ \\
\hline
\end{tabular}




\section{Discussion}

The results clearly showed that there were no significant sex-related differences in the incidence of the diseases. Nearly similar results were observed by Aggarwal et al. (1989) in India. The prevalence rate of enteropathogens found in this study is low in comparison to other findings such as $53.7 \%$ isolation by Mertens et al. (1990) in the dry zone of Sri-Lanka, and identification of $61.9 \%$ enteropathogens by Seas et al. (2000) in Peru. The variation between the prevalence rates of enteropathogens may be due to different geographic set up, socioeconomic status, environmental hygiene, water and sanitary measures, study time difference and exclusion of certain enteropathogens of diarrhoea in the study, etc.

Children are more likely than adults to die from diarrhoea because they become dehydrated more quickly. In this study, the age group distribution showed that most of the diarrhoeal episodes $(28.23 \%)$ as well as positive cases of enteropathogens $(34.31 \%)$ were prevalent in the adults of age group 20-30 years. This result is in contrast to other reports by different researchers at different times where diarrhoeal diseases have been frequently reported in children. The reason behind this is that Sukraraj Tropical Hospital (STH) is the only infectious disease hospital attending diarrhoeal patients among the adult population located in Kathmandu, Nepal and majority of patients $(82.94 \%)$ coming for laboratory diagnosis to NPHL during study period were those referred from STH.

August was found to be the month of highest incidence of diarrhoeal episode (78) and enteric isolates $(44.87 \%)$ followed by July and September in this study, which was in accordance with Pokhrel et al. (1997) that the outbreaks occur commonly from June to November each year that coincides with rainy and post-rainy seasons, the peak period being June and August suggesting favourable condition for its proliferation. The increase in rainfall, temperature and relative humidity are contributing environmental factors and also rapid contamination and transmission of organisms in huge dosage as a result of rain (Shrestha 1995). Increased frequency of diarrhoeal cases (102) and enteropathogen positive cases $(48.03 \%)$ were observed among the people who were using municipal tap water for drinking purpose. This may be due to passage of enteropathogens directly by contaminated water to the gastrointestinal tract. According to WHO (2004), $88 \%$ of diarrhoeal disease is attributed to unsafe water supply, inadequate sanitation and hygiene; and improved water supply reduces diarrhoea morbidity by $6 \%$ to $25 \%$, if severe outcomes are included. The major bacteria and parasites are discussed below.

\section{Cholera}

V. cholerae was the most prevalent isolate among enteropathogens isolated in this study. All isolated Vibrio were serogroup O1 "Inaba" and biotype "El Tor". According to Shrestha (1997), it was reported that for the last 6 years $V$. cholerae El Tor O1 Ogawa had been responsible for the endemic situation in Kathmandu, but in 1990 it was $V$. cholerae El Tor O1 Inaba. So it can be said that after 15 years later, $V$. cholerae $\mathrm{O} 1$ Inaba emerged hugely in Kathmandu. Also in India the toxigenic Inaba serotype of $V$. cholerae $\mathrm{O} 1$ biotype $\mathrm{El}$ Tor reappeared in 1998 and 1999, almost 10 years after its last dominance in Calcutta in 1989 (Garg et al. 2000).

$V$. cholerae $\mathrm{O} 1$ strains with such a periodic shift of different serotypes or serotype conversion between the Inaba and Ogawa may be a consequence of genetic reversion which is known to take place in vitro and in vivo (Colwell et al. 1995, Gangarosa et al. 1967, Stroeher et al. 1992). The genes responsible for O1 antigen biosynthesis have been designated wbe [previously known as $r f b$ ] (Reeves et al. 1996) and are localized on a 21.6-kb SacI fragment of DNA (Fallarino et al. 1997). This region is highly conserved; the only changes observed between the Ogawa and Inaba serotypes are related to a mutation in the $w b e T$ region (Yamasaki et al. 1999, Stroeher et al. 1992). According to Manning et al. 1994, the epidemic strains had undergone a serotype conversion, possibly because of immune pressure in the population. Gangarosa et al. (1967) described that the switch in O-antigen from Ogawa to Inaba is caused by selective pressure of antiOgawa antibodies and the emergence of anti-Inaba antibodies.

In the present study, the highest cholera cases were seen among the age group between 21-30 years and were predominant in male. Although children are the primary victims of cholera, the present results may be due to higher frequency of samples from adult patients. Our research found the incidence of cholera in a peak in August followed by September. This result is advocated with the preamble of Pokhrel et al. (1997) and Shrestha (1995). It is almost a regular feature now to have an 
outbreak of cholera every summer in Kathmandu valley. Vomiting, dehydration and nausea with watery or rice watery (predominantly) stool and stool passage frequency e"10 (in majority cases) were the clinical manifestations exhibited by cholera patients.

\section{Shigellosis}

Shigella still accounts for a significant proportion of bacillary dysentery in many tropical and subtropical countries (Zafar et al. 2005). As a very low number of organisms are required for causing infection, mothers not practicing proper hand washing are the main cause associated with childhood dysentery (Khan 1988, Han \& Hlaing 1989).

The pattern of serotypes found in our study was: $9.8 \%$ in S. flexneri, $4.9 \%$ in S. dysenteriae, $2.94 \%$ in $S$. boydii and $0.98 \%$ in $S$. sonnei of the total enteric isolates. This pattern of serotype finding is in consistency with the finding of Iwalokun et al. (2001) in which $S$. flexneri $(51.6 \%)$ was predominantly isolated followed by $S$. dysenteriae (17.7\%), S. boydii (17.7\%) and $S$. sonnei (13\%) respectively from diarrhoeal patients in Lagos. The predominance of S. flexneri may be due to highly infectious by the oral route, and ingestion of as few as ten organisms can cause an infection. S. flexneri and S. dysenteriae serotype 1 occur more frequently in developing countries and $S$. sonnei is more common in developed countries. Incidence of Shigella was highest in July and infection occurred mostly by consumption of direct municipal tap water. Abdominal pain, rarely fever, dehydration, nausea and vomiting were reported in the patients with shigellosis. Shigella was isolated from both loose and watery stool but in majority of the cases stools were bloody and mucoid. The major clinical manifestations of the patients infected with Shigella spp. were bloody diarrhoea, abdominal pain, anorexia, vomiting (frequently in case of $S$. sonnei \& $S$. boydii), tenesmus and fever (mostly in case of $S$. dysenteriae type 1 and S. flexneri), in a study conducted by Bhattacharya $e t$ al. (1994) in Calcutta during 1990-1992. Bloody diarrhoea is usually a sign of invasive enteric infection that carries a substantial risk of serious morbidity and death.

\section{Salmonellosis}

Salmonellosis is a bacterial infection that generally affects the intestinal tract and occasionally the bloodstream. It is one of the more common causes of diarrhoeal illness. Salmonella spp., a common cause of food poisoining is usually associated with food borne infections in contrast to the enteric fever caused by $S$. typhi and $S$. paratyphi which is basically waterborne infection. In higher-risk groups, Salmonella is more likely to invade beyond the gastrointestinal tract and cause bacteriamia. From there the bacteria can spread deeper into the body and cause more severe symptoms. Infection rate of Salmonella is higher in industrialized countries and cities where canned foods are mostly used. Non-typhoidal Salmonella are one of the key etiological agents of diarrhoeal disease.

In this study, out of a total of $9(8.82 \%)$ Salmonella isolates, 2(1.96\%) were S. typhi and S. typhimurium each and the rest $(4.9 \%)$ were Salmonella species (polyvalent A-S positive). Salmonella was predominant among male and in the patients of age group 10-20; incidence was peaked in June and abdominal pain, fever, rarely vomiting, dehydration nausea and stool passage frequency with e" 10 per day were reported as the main clinical features of salmonellosis. Salmonella were isolated from loose or watery stools of diarrhoeal patients with some cases having blood and mucus. People using direct tap water suffered frequently than people using boiled or filtered water.

Koplan et al. (1979) suggested that the best clinical predictor of positive stool culture (Salmonella and Shigella) was the combination of more than 24 hours fever and either blood in the stool or abdominal pain with nausea and vomiting.

In Nepal majority of Salmonella spp. isolated from diarrhoeal patients in former studies were also $S$. typhimurium as published by researchers. It was the most common isolate $(45.7 \%)$ in diarrhoeal children having less than 5 years age in Iran (Farhoudi et al. 1990) followed by S. havana (30.9\%), S. typhi (2.9\%) and S. larochelle $(2.4 \%)$.

\section{Parasites}

Intestinal parasitosis leading to diarrhoeal and dysenteric manifestations is one of the major public health problems in Nepal. Overall, the intestinal protozoal parasitosis and intestinal helminthiasis rank the second and the fourth respectively in the list of top ten diseases of Nepal. However, a hospital based study has shown a declining trend of the diseases during a period of one decade (1985-1994) (Rai et al. 2004). The study was also undertaken to review the prevalence and type of parasitic infestations. The stools collected from patients were screened by routine microscopic examination and cyst 
or trophozoites of protozoa and ova of helminthes positive stool were recorded. Microscopic examination of stool samples is the gold standard for diagnosis of parasites. The finding of low number of parasites in our study or temporary disappearance of cysts and ova from stools may be due to intermittence of parasitic cycle and use of different medication which may give false negative results and poor diagnostic sensitivity of the stool microscopy or limitation to direct stool microscopy only. Winiecka-Krusnell $\mathrm{J}$ and E. Linder (1995) suggested that the isolation of parasites could be detected more frequently using antibody marker for samples that contained low number of parasites which are not detected by conventional microscopy. Immunoflorescent staining technique in conjugation with microscopic examination improved the isolation rate.

From the results obtained in this study, it can be concluded that many people of different ages are suffering from diarrhoea in the rainy season in Kathmandu. Both bacteria and parasites are prevalent among diarrhoea patients. Diarrhoeal episodes could be decreased significantly by implementing simple strategies, such as better waste and water management and hygiene education. Increasing antibiotic resistance against commonly prescribed drugs signify that treatment options have become difficult in cases of severe diarrhoea. The results indicate a continuing need for resistance surveillance and rational use of antimicrobial agents to reduce the multiresistant strains.

\section{Acknowledgement}

We are thankful to Ms. Palpasa Kansakar and Mr. Gokarna Raj Ghimire for their kind help.

\section{References}

Aggarwal, P., K.K. Khanna and S. Kumar. 1989. Cholera gastroenteritis amongst children in Delhi. Indian Journal of Paediatriac 56: 93-96.

Bhattacharya, M. K., S.K. Bhattacharya, M. Paul, D. Dutta, P. Dutta, H. Kole, D. De, A.R. Ghosh, P. Das and G.B. Nair. 1994. Shigellosis in Calcutta during 1990-1992: antibiotic susceptibility pattern and clinical features. Journal of Diarrhoeal Disease Research 12(2): $121-4$

Bista, M.B., K. Shrestha and U.N. Devkota. 1993. Gastroenteritis, encephalits, meningitis and kalazar. Epidmiology Division, Ministry of Health, Teku, Kathmandu. pp. 3-27.
Colwell, R.R., A. Huq, M.A.R. Chowdhury, P.R. Brayton and B. Xu. 1995. Serogroup conversion of Vibrio cholerae. Canadian Journal of Microbiology 41: 946-950.

Fallarino, A., C. Mavrangelos, U.H. Stroeher and P.A. Manning. 1997. Identification of additional genes required for O-antigen biosynthesis in Vibrio cholerae O1. Journal of Bacteriology 179: 21472153.

Farhoudi, M., A. Ali, M. Ratouli, A. Jafari, M.A. Bhavar, M. Parsi and F. Malekzadeli. 1990. Antimicrobial drug resistance and resistance factor transfer among clinical isolates of salmonellae in Iran. Journal of Infectious Diseases 22(2): 197-203.

Gangarosa E.J., A. Sanati, H. Saghari and J.C. Feeley. 1967. Multiple serotypes of Vibrio cholerae isolated from a case of cholera. Lancet 1: 646-648.

Han, A.M. and T. Hlaing. 1989. Prevention of diarrhoea and dysenteries by hand washing. Trans R. Soc. Trop. Med. Hyg. 83: 128-31.

Iwalokun, B.A., G.O. Gbenle, S.I. Smith, A. Ogunledun, K.A. Akinsinde and E.A. Omonigbehin. 2001. Epidemiology of shigellosis in Lagos, Nigeria: trends in antimicrobial resistance. Journal of Health, Population and Nutrition 19(1)183-190.

Khan, M.M., J. Iqbal, A. Ghafoor and M.I. Burney. 1988. Aetiologic agents of diarrhoeal diseases in hospitalised children in Rawalpindi, Pakistan. Journal of Diarrhoeal Disease Research 6(3-4): 228-31.

Koplan, J.P., H.V. Fineberg, M.J.V. Ferraro and M.I. Roseberg. 1979. Value of examination of feacal leucocytes in early diagnosis of shigellosis. Am. J. Trop. Med. Hyg. 28: 1031-5.

Manning, P.A., U.H. Stroeher and R. Morona. 1994. Vibrio cholerae and cholera: molecular to global perspectives. In: Molecular basis for O-antigen biosynthesis in Vibrio cholerae O1: Ogawa-Inaba switching. (Eds K. Wachsmuth, P. A. Blake, and O. Olsvik). ASM Press, Washington D.C. pp. 77-94.

Mertens, T.E., R. Wijenayake, M.R. Pinto, J.S. Peiris, M.D. Wijesundera, N.B. Eriyagama, K.G. Karunarathne and L.R. Ranaweera. 1990. Microbiological agents associated with childhood diarrhoea in the dry zone of Sri Lanka. Tropical Medical Parasitology 4(1):115-20.

Pokhrel, B.M., B.R. Prasai, T. Kubo, A.P. Sharma, N.R. Tuladhar, S.P. Shrestha, C. Gurung and H. Ohara. 1997. Bacteriology of diarhoea in Nepal. Journal of Institute of Medicine 19: 128-13.

Rai, S.K. 2004. Parasitic diseases in Nepal. In: Food-Borne Helmintheasis in Asia. Asian parasitology. The federation of Asian Parasitologists. (Eds. Arizono K, Chai J, Nawa Y and Takahansi Y). Southeast Asian Journal of Tropical Medicine and Public Health 35: 19-23 
Reeves, P.R., M. Hobbs, M.A. Valvano, M. Skurnik, C. Whitfield, D. Coplin, N. Kido, J. Klena, D. Maskell, C.R.H. Raetz and P. D. Rick. 1996. Bacterial polysaccharide synthesis and gene nomenclature. Trends in Microbiology 4: 495-503.

Seas, C., M. Aragon and G. Eduardi. 2000. Survillence of bacterial pathogens associated with acute diarrhoea in Lima, Peru. International Journal of Infectious Diseases 4(2): 96-99.

Sherchand, J. B., S. Larsson and M.P. Shrestha. 1996. Intestinal parasites in children and adults with and without abdominal discomfort from the Kathmandu area of Nepal. Tropical Gastroenterol. 17: 15-22.

Shrestha, K.D. 1994. Acute diarrhoeal cases in Nepal, 1993. An epidemiological review: Journal of Nepal Medical Association 32(110) 68-85.

Shrestha, K.D. 1995. Acute diarrhoeal cases in Nepal during outbreak season. An epidemiological review. Journal of Nepal Medical Association 33: 219-231.

Stroeher, U.H., L.E. Karageorgos, R. Morona and P.A. Manning. 1992. Serotype conversion in Vibrio cholerae O1. Proceeding of National Academy of Science USA 89: 2566-2570.

Sunoto, P. 1982. Diarrhoeal problems in Southeast Asia. Southest Asian Journal of Tropical Medical Health 13(3): 306-18.
WHO. 1992. Reading on diarrhoea: A student manual ( $1^{\text {st }}$ Ed.). Geneva.

WHO. 2004. The health hazards from poor water, sanitation and hygiene behaviour. Geneva.

Winiecka-Krusnell, J. and E. Linder 1995. Detection of Giardia lamblia cysts in stool samples by immunofluorescence using monoclonal antibody. European Journal of Clinical Microbiology and Infectious Diseases 14(3): 218-222.

Wolf, D.C. and R.A. Giannella. 1993. Antibiotic therapy for bacterial enterocolitis: a comprenhensive review. American Journal of Gastroenteritis 88: 1667-1683.

Yamasaki, S., Garg S, Nair G B and Y. Takeda. 1999. Distribution of Vibrio cholerae $\mathrm{O} 1$ antigen biosynthesis genes among O139 and other nonO1 serogroups of Vibrio cholerae. FEMS Microbiol. Lett 179: 115-121.

Zafar, A., N. Sabir and Z.A. Bhutta. 2005. Frequency of isolation of Shigella serogroups/serotypes and their antimicrobial susceptibility pattern in children from slum areas in Karachi. Journal of Pakistan Association 55(5): 184-8. 